\title{
Research on the Complexity and Chaos Control about a Closed-Loop Supply Chain with Dual-Channel Recycling and Uncertain Consumer Perception
}

\author{
Junhai Ma ${ }^{\mathbb{D}},{ }^{1}$ Hao Ren $\mathbb{D}^{1},{ }^{1}$ Miao Yu ${ }^{\mathbb{D}},{ }^{1}$ and Meihong Zhu $\mathbb{D}^{1,2}$ \\ ${ }^{1}$ College of Management and Economics, Tianjin University, Tianjin 300072, China \\ ${ }^{2}$ Zhejiang University of Water Resources and Electric Power, Zhejiang 310018, China \\ Correspondence should be addressed to Miao Yu; miaoyu161028@126.com
}

Received 12 May 2017; Accepted 7 September 2017; Published 11 January 2018

Academic Editor: Julio Blanco-Fernández

Copyright (C) 2018 Junhai Ma et al. This is an open access article distributed under the Creative Commons Attribution License, which permits unrestricted use, distribution, and reproduction in any medium, provided the original work is properly cited.

\begin{abstract}
According to game theory, chaotic dynamics theory, and complexity based on the literature review about the pricing strategy and recycling channels of the closed-loop supply chain, on the background of the widespread hybrid recycling channels in Chinese electronic products market, this paper builds a closed-loop supply chain model with dual-channel recycling composed of one manufacturer and one third-party. We assume that the consumer perception towards the remanufacturing products is uncertain and limited. We carry on theoretical analysis of this model and perform numerical simulations from the perspective of entropy theory. Results show that a precipitous speed of recycling price adjustment of the manufacturer or the third-party will both lead the system into a chaotic state and cause the entropy of the system to increase. The system is sensitive to initial conditions in this chaotic condition. Focusing on the harmful effects of chaotic system, we introduce adjustment parameter to efficiently control the chaos. The results have a strong reference value to practical problems, so it has a great value in both theory and application.
\end{abstract}

\section{Introduction}

Along with the economy's continuous development, waste products have attracted more and more attention. Growth in the living standard and people's requirement for the quality of life gives rise to a lot of products being wasted before the end of their life cycle. A mass of waste products is not only tremendously harmful to our environment, but also a great waste of resources. So how to recycle and reuse waste products reasonably and effectively becomes a widely concerned problem around the world. Many countries have introduced policies, laws, and regulations in order to increase repetitive use rate of raw material. Nowadays, more and more companies bring closed-loop supply chain management into the enterprise strategic management.

In recent years, international and domestic academics have carried on extensive researches about closed-loop supply chain, mainly focused on pricing of new products and used products in closed-loop supply chain, the recycling channel in closed-loop supply chain, coordination, and contracts for profit sharing in closed-loop supply chain. Many scholars have established a closed-loop supply chain model from the perspective of operational research and game theory. Liang et al. [1] held the view that new products and reproducing products have different prices in market and the retail price of remanufacturing products follows geometric Brownian motion. Based on this opinion, they introduced a model to connect recycling price with selling price. Ketzenberg [2] analyzed the value of information in a capacity limited closed-loop supply chain system. Salema et al. [3] built a closed-loop supply chain with multiproduct and multistage and put forward the optimal product flow route design by the way of network simulation of product flow in reverse logistics. Kannan et al. [4] performed a simulation of the recycling of waste battery using genetic algorithm and built a closed-loop supply chain network model with multilevel and multiproduct, which solved the decision problem of material purchasing, producing, distribution, recycling, and processing. Sheu and Lin [5] presented a multiobjective programming model including the network configuration 
cost, the profits, and consumer satisfaction level, considering the recovery rate and subsidies from the government. Beyond that they also take the potential risk-oriented costs into account. Lu et al. [6] introduced service and consumers switching to repetitive manufacturing and studied the influence of service level on profits. They put forward and proved the importance of the factor of service. Chen and Chang [7] discussed under what conditions Original Equipment Manufacturer (OEM) should participate in the recycling and reproducing. The analysis result indicates that OEM's strategic decision mainly depends on the remanufacturing cost and the competition intensity between OEM recycling and the third-party recycling. Huang et al. [8] discussed a closed-loop supply chain with a retailer and a third-party who specialized in recycling waste products competitively recycling used products and compared the optimal decisions in dual-channel recycling and single-channel recycling. Maiti and Giri [9] considered a supply chain with a manufacturer selling new products and remanufactured products through a retailer and recycling waste products via a third-party. They assumed the demand is connected with the quality of new and reproducing products and investigate Nash game and Stackelberg game under different channel powers. He [10] studied the closed-loop supply chain with uncertain demand and uncertain supply, respectively, and analyzed the optimal decisions of recycling price and remanufacturing under centralized decision-making and decentralized decision-making. They also discussed the influence of the uncertainty level of demand and supply on each variable in the closed-loop supply chain. Zu-Jun et al. [11] built a three-stage supply chain composed of one manufacturer, one retailer, and two recycle bins and discussed the influence of different cooperation structure on decisions in closed-loop supply chain.

However, most of these studies on closed-loop supply chains are static models from the perspective of operational research methods and optimization methods. But for the closed-loop supply chain, which needs to consider the characteristics of the forward channel and reverse channel, the static model can not reflect the actual situation of sales. As a complex system, it is necessary to establish the model from the perspective of complex characteristics and entropy.

Some researchers connect the complex dynamics theory with supply chain and analyze the dynamic feature of the system. Elsadany [12] built a duopoly game model based on bounded rationality with delayed decision and analyzed its complexity and the local stability of equilibrium point. It turned out that companies with delayed bounded rationality have more opportunities to reach the Nash equilibrium. Guo and Ma [13] presented a closed-loop supply chain with a manufacturer and a retailer; the retailer recollects the waste products from consumers. They discussed the complex dynamic phenomena of the system and the influences of the system parameters on the base of the manufacturer being the leader in the Stackelberg game. Ma and Guo [14] built a closed-loop supply chain with two competitive retailers and a manufacturer based on waste household appliance market in China. They found that precipitous speed of price adjustment would not only cause the chaotic state but also deteriorate and invalidate the competition. Xie and Ma [15] studied the competitive behavior in close-loop supply chain with thirdparty recycling using the theory of complex system.

In recent years, based on the entropy theory, some scholars found that we can analyze the dynamic characteristics of the system effectively by the magnitude of entropy values. There are some researches who connected entropy with management and economics. In Ma and Si [16] research, entropy was used to predict the financial time series; they drew the conclusion that financial forecasts of foreign exchange currency in low entropy regions have a slight advantage in trading, and it is very difficult to achieve the predicting time series in high entropy regions. Han et al. [17] established a hydropower duopoly game with two time delays and analyzed the influence of delay parameter on the entropy and dynamic features of the system.

On the basis of previous studies on the static model, we draw on the application of complex system theory and entropy theory in management; we build a closed-loop supply chain with dual-channel recycling based on the real situation of Chinese electronic products market in this paper. We analyze the complex systems of closed-loop supply chains from a dynamic perspective. The structure of this paper is as follows: the second part is the assumptions, the model structure, symbol explanation, and model analysis; the third part describes the dynamic characteristics of the system based on numerical simulation; the fourth part introduces a method to control chaos; and the summary is made in the fifth part.

\section{Basic Model and Analysis}

2.1. Assumption. There are mainly three kinds of recycling ways in closed-loop supply chain-recycled by the manufacturer, the retailer, and the third-party. With the intensity of market competition, recollecting used products through hybrid channels emerged; that means different recycling modes collect the used products simultaneously and competitively. In this paper, we explore a closed-loop supply chain with dual-channel recycling. The manufacturer and the thirdparty recollect the used product at the same time. Figure 1 shows the recycling method in this paper.

According to this closed-loop supply chain system, we made the following assumptions.

(1) The recycling function is linear. The quantity of recollecting used products is only affected by the recycling prices of itself and its competitor (the number of the collecting is increasing function of its collecting price), ignoring the influence of environment, service and consumption level, and so on.

(2) The unit cost of manufacture's recycling and remanufacturing used products is less than the unit cost of manufacturing new products, which means that the manufacturer chooses recycling which is profitable.

(3) The collecting capability and remanufacturing capability are unlimited. In order to simplify the problem, we assume that all the collecting products can be remanufactured. 


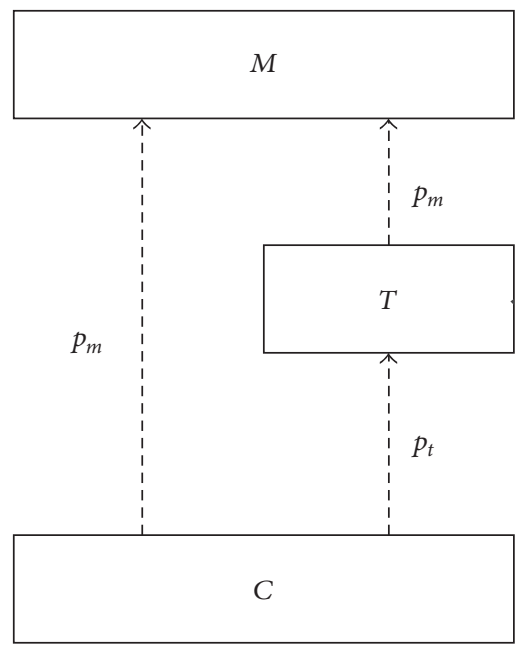

FIGURE 1: Recycling method of the closed-loop supply chain with dual-channel recycling.

(4) Consumer perception of the remanufactured products is inferior to that of the new products. In this paper, we denote consumer perception by the amount of remanufactured products which consumers are willing to buy in one time period.

(5) The manufacture and the third-party are both independent decision makers in this closed-loop supply chain; they make respective decisions in discrete periodic points $t=0,1,2, \ldots$, to maximize their own profits. Due to the constraints of market conditions, decision makers cannot be fully aware of others' behaviors and the market conditions, so they can only make bounded rational decisions.

\subsection{Symbol Description and Modeling}

$c_{l}$ is unit cost when the manufacturer or the thirdparty recycles the used products from consumers.

$\Delta$ is unit cost saving when the manufacturer remanufacture a used product instead of manufacturing a new product.

$p_{t}$ is unit recycling price of the third-party from consumers, decision variable of the third-party.

$p_{m}$ is unit recycling price of the manufacturer from consumers and the third-party, decision variable of the manufacturer.

So the recycling quantities of the manufacturer and the third-party are, respectively, expressed as $Q_{m}$ and $Q_{t}$.

$$
\begin{aligned}
Q_{m} & =a_{1}+b p_{m}-c p_{t} \\
Q_{t} & =a_{2}+b p_{t}-c p_{m} .
\end{aligned}
$$

In the formula above, $a_{1}$ and $a_{2}$ stand for the quantity of used products which consumers voluntarily return when the unit recycling price of the manufacturer or the thirdparty is equal to zero, namely, consumers' environmental awareness. $b$ indicates the consumers' recycling price sensitivity coefficient. $c$ denotes the recycling price competition coefficient between the manufacturer and the third-party, and the coefficients should meet the condition $b \geq c$ based on the actual situation.

We use $Q$ to signify the total quantity of recollected products from the manufacturer and the third-party; then we can tell that $Q=Q_{m}+Q_{t}$.

Because the consumer perception towards the remanufactures products is uncertain and limited compared to the new products, a parameter $d$ which obeyed uniform distribution in the interval $\left[h-h_{1}, h+h_{1}\right]$ can be used to show the quantity of remanufactured products consumers are willing to buy in period $t$. The distribution function is $F(d)$, and probability density function is $f(d)=1 / 2 h_{1}$. If the quantity of total recollected products exceeds $d$, only $d$ can be sold to consumers, and the part beyond $d$ would be lost. On the other hand, if $d$ is less than $Q$, all of the remanufactured products can be sold out. But in this case, some consumers who are willing to buy remanufactured products in the beginning have to buy new products instead or give up buying behavior, which will bring losses to the manufacturer as well. We use $s$ to denote the unit loss of the manufacturer due to the lack of recycling products.

The profit models of the manufacturer and the third-party can be written as follows:

$$
\begin{aligned}
& \pi_{M}= \begin{cases}\left(\Delta-p_{m}-c_{l}\right) * d-\left(p_{m}+c_{l}\right)(Q-d) & Q \geq d \\
\left(\Delta-p_{m}-c_{l}\right) * Q-s *(d-Q) & d \geq Q\end{cases} \\
& \pi_{T}=\left(p_{m}-p_{t}-c_{l}\right) Q_{t} .
\end{aligned}
$$

The expected profit function of the manufacturer is

$$
\begin{gathered}
E\left(\pi_{M}\right)=\int_{Q}^{h+h_{1}}\left(\left(\Delta-p_{m}-c_{l}\right) * Q-s *(d-Q)\right) \\
* f(d) d(d)+\int_{h-h_{1}}^{Q}\left(\left(\Delta-p_{m}-c_{l}\right) * Q-s\right.
\end{gathered}
$$




$$
\begin{aligned}
& *(d-Q)) * f(d) d(d)=\frac{1}{4 h_{1}}\left(\left(a_{1}+a_{2}-h-h_{1}\right.\right. \\
& \left.+(b-c)\left(p_{m}+p_{t}\right)\right)\left(\left(h+h_{1}\right) * s+\left(a_{1}+a_{2}\right)\right. \\
& *\left(2 c_{l}+2 p_{m}-s-2 \Delta\right)+(b-c) *\left(p_{m}+p_{t}\right) \\
& \left.*\left(2 c_{l}+2 p_{m}-s-2 \Delta\right)\right)-\left(a_{1}+a_{2}-h+h_{1}\right. \\
& \left.+(b-c) *\left(p_{m}+p_{t}\right)\right) \\
& \cdot\left(2\left(c_{l}+p_{m}\right)\left(a_{1}+a_{2}+(b-c)\left(p_{m}+p_{t}\right)\right)\right. \\
& \left.\left.-\left(a_{1}+a_{2}+h-h_{1}+(b-c)\left(p_{m}+p_{t}\right)\right) \Delta\right)\right) .
\end{aligned}
$$

As mentioned above, the manufacturers' decision variable is $p_{m}$, the third-party's decision variable is $p_{t}$, and the marginal profit functions are

$$
\begin{aligned}
\frac{\partial \pi_{M}}{\partial p_{m}}= & \frac{1}{2 h_{1}}\left(a_{1}+a_{2}\right)\left(-2 h_{1}-(b-c)(s+\Delta)\right) \\
& -(b-c) *\left(2 c_{l} h_{1}\right) \\
& +h_{1}\left(4 p_{m}+2 p_{t}-(s+\Delta)\right) \\
& -\left(h-(b-c)\left(p_{m}+p_{t}\right)\right)(s+\Delta) \\
\frac{\partial \pi_{T}}{\partial p_{t}}= & -a_{2}+c p_{m}+b\left(-c_{l}+p_{m}-2 p_{t}\right) .
\end{aligned}
$$

Because of the complexity of the market, the manufacturer and the third-party cannot acquaint entirely and predict accurately the true information of the market and the complete information of other decision makers. Therefore, all of the decision makers in the market are based on bounded rationality model. The decisions in period $t+1$ under bounded rational hypothesis are

$$
\begin{aligned}
& p_{m}(t+1)=p_{m}(t)+g_{1} p_{m}(t) \frac{\partial \pi_{M}}{\partial p_{m}} \\
& p_{t}(t+1)=p_{t}(t)+g_{2} p_{t}(t) \frac{\partial \pi_{T}}{\partial p_{t}}
\end{aligned}
$$

In the formula above, $g_{i}>0(i=1,2)$, respectively, stands for the manufacturer's adjusting speed of $p_{m}$, and the third-party's adjusting speed of $p_{t}$. We can get the twodimensional discrete dynamic equation as follows:

$$
\begin{aligned}
& p_{m}(t+1)=p_{m}(t)+g_{1} p_{m}(t) \frac{1}{2 h_{1}}\left(a_{1}+a_{2}\right)\left(-2 h_{1}\right. \\
& \quad-(b-c)(s+\Delta))-(b-c) *\left(2 c_{l} * h_{1}\right. \\
& \quad+h_{1}\left(4 p_{m}+2 p_{t}-(s+\Delta)\right) \\
& \left.\quad-\left(h-(b-c)\left(p_{m}+p_{t}\right)\right)(s+\Delta)\right) \\
& p_{t}(t+1)=p_{t}(t)+g_{2} p_{t}(t)\left(-a_{2}+c p_{m}\right. \\
& \left.\quad+b\left(-c_{l}+p_{m}-2 p_{t}\right)\right) .
\end{aligned}
$$

2.3. Model Analysis. After the decision variables' continuously adjustment for several periods, the dynamical system above can achieve an equilibrium state. In this state, each of the decision-making parties may not increase their profit by changing the value of the decision variable. So the value of the decision variable would not be adjusted any more, and every party achieves the profit maximization. The equilibrium solution of our system is $R_{1} \sim R_{4}$.

$$
\begin{aligned}
& R_{1}(0,0) \\
& R_{2}\left(\frac{\left(a_{1}+a_{2}\right)\left(-2 h_{1}-(b-c)(s+\Delta)\right)-(b-c)\left(2 h_{1} c_{l}-\left(h+h_{1}\right)(s+\Delta)\right)}{(b-c)\left(4 h_{1}+(b-c)(s+\Delta)\right)}, 0\right) \\
& R_{3}\left(0,-\frac{a_{2}+b c_{l}}{2 b}\right) \\
& R_{4}\left(p_{m}{ }^{*}, p_{t}{ }^{*}\right) .
\end{aligned}
$$

In $R_{4}$,

$$
\begin{aligned}
& p_{m}^{*}=\frac{\left(-2 a_{1} b-a_{2}(b+c)\right)\left(2 h_{1}+(b-c)(s+\Delta)\right)+b(b-c)\left(2\left(h+h_{1}\right)(s+\Delta)+c_{l}\left(-2 h_{1}+(b-c)(s+\Delta)\right)\right)}{(b-c)\left(3 b^{2}(s+\Delta)+2 b\left(5 h_{1}-c(s+\Delta)\right)-c\left(-2 h_{1}+c(s+\Delta)\right)\right)} \\
& p_{t}^{*}=-\left(a_{1}(b+c)\left(2 h_{1}+(b-c)(s+\Delta)\right)+2 a_{2}\left(-c h_{1}+b^{2}(s+\Delta)+b\left(3 h_{1}-c(s+\Delta)\right)\right)+(b-c) b^{2} c_{l}(s+\Delta)\right. \\
& \left.\quad+c\left(2 c_{l} h_{1}-\left(h+h_{1}\right)(s+\Delta)\right)+b-\left(h+h_{1}\right) *(s+\Delta)+c_{l}\left(6 h_{1}-c(s+\Delta)\right)\right) \\
& \quad \times\left((b-c)\left(3 b^{2}(s+\Delta)+2 b\left(5 h_{1}-c(s+\Delta)\right)-c\left(-2 h_{1}+c(s+\Delta)\right)\right)\right)^{-1} .
\end{aligned}
$$


We can find that among the 4 equilibrium solutions above, only $R_{4}$ is completely nonzero, while the other three are partly or entirely zero. Decision makers obviously do not allow their decision variable to be zero in economics, which means that only $R_{4}$ is stable among the 4 equilibrium solutions and $R_{1} \sim R_{3}$ is unstable. We can verify it by solving the Jacobian matrix of each equilibrium solution.

The Jacobian matrix of our system can be written as follows:

$$
=\left(\begin{array}{cc}
J_{11} & -\frac{(b-c) g_{1} p_{m}\left(2 h_{1}+(b-c)(s+\Delta)\right)}{2 h_{1}} \\
(b+c) g_{2} p_{t} & J_{22}
\end{array}\right),
$$

where

$$
\begin{aligned}
J_{11} & =1-\frac{1}{2 h_{1}}\left(g _ { 1 } \left(a_{1}\left(2 h_{1}+(b-c)(s+\Delta)\right)+a_{2}\left(2 h_{1}\right.\right.\right. \\
& +(b-c)(s+\Delta))+(b-c)\left(2 c_{1} h_{1}\right. \\
& +h_{1}\left(8 p_{m}+2 p_{t}-s-\Delta\right) \\
& \left.\left.\left.-\left(h-(b-c)\left(2 p_{m}+p_{t}\right)\right)(s+\Delta)\right)\right)\right) \\
J_{22} & =1+g_{2}\left(-a_{2}+c p_{m}+b\left(-c_{l}+p_{m}-2 p_{t}\right)-2 b p_{t}\right) .
\end{aligned}
$$

We calculate the Jacobian matrix of the 4 equilibrium solutions next to judging its stability according to the magnitude of the eigenvalues. If a nonzero eigenvalues are greater than 1 , then this equilibrium solution is unstable.

The characteristic equation takes the form

$$
\begin{aligned}
F(\lambda) & =|\lambda E-J| \\
& =\lambda^{2}-\left(J_{11}+J_{22}\right) \lambda+\left(J_{11} J_{22}-J_{12} J_{21}\right) .
\end{aligned}
$$

According to the Jury criterion, the coefficients of the characteristic polynomial should satisfy the following conditions:

$$
\begin{array}{r}
-\left(J_{11}+J_{22}\right)+\left(J_{11} J_{22}-J_{12} J_{21}\right)+1>0 \\
\left(J_{11}+J_{22}\right)+\left(J_{11} J_{22}-J_{12} J_{21}\right)+1>0 \\
J_{11} J_{22}-J_{12} J_{21}<1 \\
J_{11}+J_{22}>0 .
\end{array}
$$

In theory, we can get the system stable by solving the condition, but our system is too complicated; we will analyze its dynamic characteristics by numerical simulation.

\section{Numerical Simulation}

Nowadays, numerical simulation is widely used in the representation of complex dynamic system. In this paper, we use MATLAB to perform numerical simulation and analysis of its dynamics feature. We take the parameter values as follows: $a_{1}=0.2, a_{2}=1, c_{l}=0.1, b=1, c=0.4, \Delta=8, s=1$, $h=4$, and $h_{1}=1$ Because these parameters are the necessary factors for manufacturers to fulfill their basic production activity and the analyzing aim is to find out the threshold of adjustment parameters while it will affects system status in the following study. So we pick out these basic parameters as the fundamental precondition of further research.

In this section, we will demonstrate the dynamic characteristics of the system in three ways, including the bifurcation diagram, the largest Lyapunov exponent, and the chaotic attractors. Entropy can also effectively measure the degree of chaos and disorder in the system. If the entropy value is larger, the system would be in a more chaotic state. If the energy is absolutely uniformly distributed in the space, the entropy of the system would maximize. We will discuss the impacts of the decision variables' adjustment on the system's stability and analyze the sensitivity to initial value.

3.1. Chaos and Bifurcation Phenomenon. Bifurcation diagram is an intuitive approach to show the dynamic characteristics of the system with one parameter changed and other parameters unchanged. Ceteris paribus, we assume that $g_{2}$ is fixed at 0.01 and $g_{1}$ changes from 0.22 to 0.4 ; Figure 2(a) shows the bifurcation diagram of the manufacturer's recycling price $p_{m}$ when $g_{1}$ is at [0.22, 0.4]. We can see that when $g_{1}$ increases from 0.22 to $0.302, p_{m}$ stays stable at 2.331. After several times' game, the first bifurcation occurs, and the system turns into stable cycles of period 2 when $g_{1}$ increases to 0.302 ; when $g_{1}$ changes from 0.36 to 0.375 , the system is stable cycles of period 4; when $g_{1}$ is between 0.375 and 0.38 , the system shows stable cycles of period 8; when $g_{1}$ is greater than 0.38 , the system gets into chaos, It means that as $g_{1}$ rises, the value of the recycling price $p_{m}$ presents from one certain value to two values and finally becomes multiple values. From the perspective of information theory, the uncertainty of the recycling price $p_{m}$ increases, so it will lead to the result that companies will need to gather more information to make decisions and the information entropy of the system increases.

The variation diagram of the third-party recycling price $p_{t}$ has been shown in Figure 2(b) when $g_{2}$ is fixed at 0.01 and $g_{1}$ changes from 0.22 to 0.4 . Similar to the bifurcation diagram of $p_{m}, p_{t}$ is stable at the value of 1.086 when $g_{1}$ increases from 0.22 to 0.302 . The first bifurcation also occurs when $g_{1}$ is at 0.302 , and, after cycles $2,4,8$, and so on, the system is gradually into the chaotic state. It also confirms that the entropy of the system is increasing as $g_{1}$ is increasing.

From the analysis above, we can find that when the adjustment speed $g_{1}$ is oversized, the uncertainty of the decision variables will increase obviously, which will cause the entropy reach to be a very large value; the market situation will get complex and the decision of decision makers will become complicated. So we suspect that the profit of decision makers will also be influenced. Figures 2(a) and 2(b) show the variation diagram of the profits of the manufacture and the third-party with respect to $g_{1}$, respectively. As we just predicted, the profits of the manufacture and the third-party also stay stable at 17.21 and 1.321 when $g_{1}$ is smaller than 0.302 ignoring error. The profits will go into chaotic state when $g_{1}$ is bigger than 0.38 . Therefore, we can reach the conclusion 


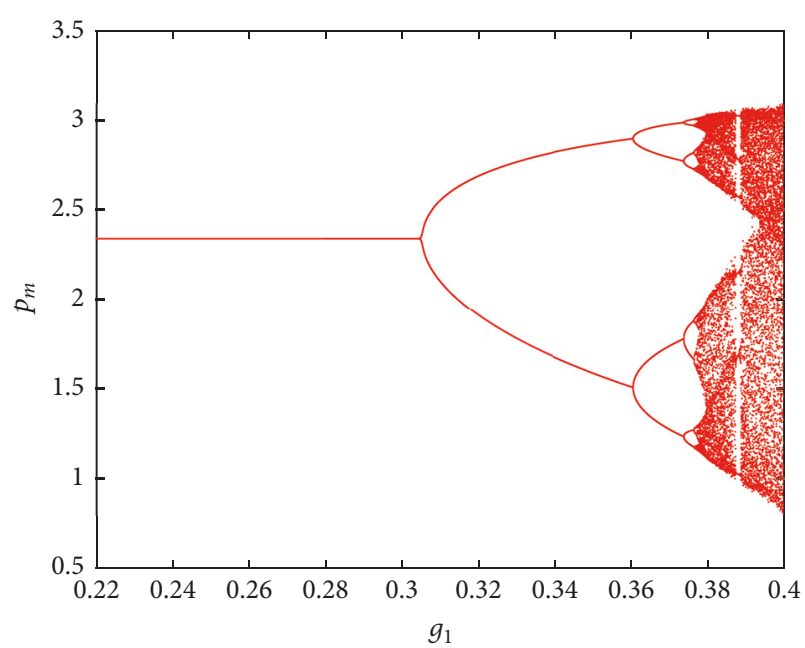

(a)

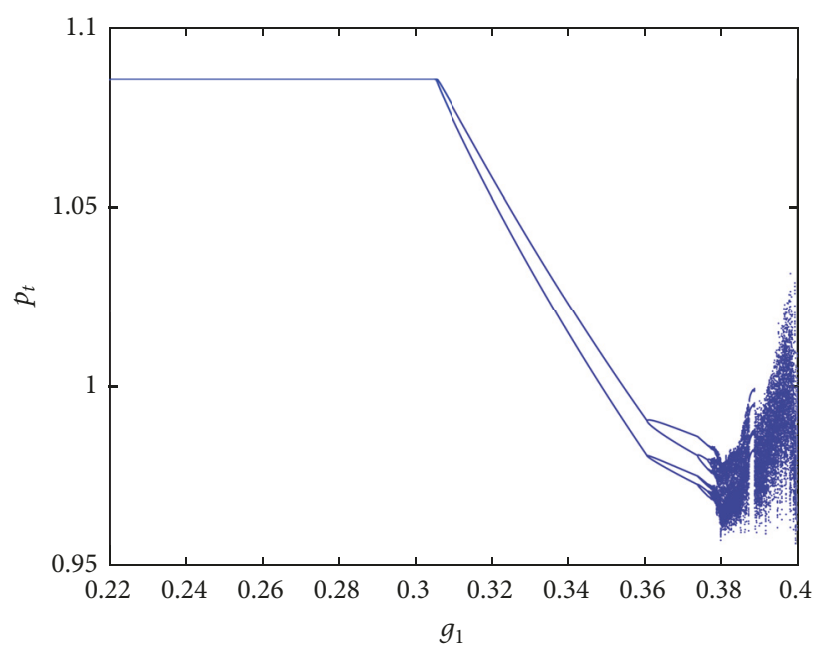

(b)

FIGURE 2: (a) Bifurcation diagram of $p_{m}$ with respect to $g_{1}$ when $g_{2}=0.01$, (b) bifurcation diagram of $p_{t}$ with respect to $g_{1}$ when $g_{2}=0.01$.

that the oversize of $g_{1}$ will not only complicate the decisionmaking, but also impact the profits of the manufacture and the third-party.

When keeping other parameters fixed, we assume that $g_{1}$ is fixed at 0.22 and $g_{2}$ changes from 0.45 to 0.587 ; Figure 4(a) shows the bifurcation diagram of the manufacturer's recycling price $p_{m}$ when $g_{1}$ is at $[0.45,0.587]$. We can see that when $g_{1}$ increases from 0.45 to $0.523, p_{m}$ stays stable at 2.337 . After multigame, the adjustment speed of the third-party's recycling price is beyond a certain level, which will prompt the system into chaos. For the third-party, the adjustment speed of the decision variable determines the length of time to achieve its optimal decision, but the increasement of adjustment parameter may bring chaos of the whole recycling system. Under chaotic state, both the manufacturer and the third-party cannot achieve a stable decision. We can find that as $g_{2}$ rises, the value of the recycling price $p_{m}$ changes from one certain value to innumerable values. From the perspective of comentropy, the increasement of the uncertainty of $p_{m}$ will result in the fact that we can be able to make the situation clear only by gathering more information. In other words, we can also say that the information entropy of the system increases.

Figure 4(b) delivers the similar information about the influence of $g_{2}$ on the stability of the third-party's recycling price. When $g_{2}$ increases from 0.45 to $0.523, p_{t}$ can maintain stability at the value of 1.09 , but if $g_{2}$ continues to increase beyond 0.523 , the system will step into chaos, which also verifies that the information entropy of the system is increasing along with the growth of $g_{2}$.

Just like the suspicion that the profit of decision makers will be influenced by the adjustment parameters, we get Figures 5(a) and 5(b) which show the variation diagram of the profits of the manufacture and the third-party with respect to $g_{2}$, respectively. From the two pictures we can find that the profits of the manufacture and the third-party also stay stable at 17.24 and 1.33 when $g_{2}$ is smaller than 0.532 ignoring error, and they will go into chaotic state when $g_{2}$ is bigger than 0.532 . Therefore, we can reach the conclusion that the oversize of $g_{2}$ will not only complicate the decision-making, but also impact the profits of the manufacture and the third-party.

Furthermore, we make $g_{1}$ and $g_{2}$ change at the same time; the changes of $p_{m}$ and $p_{t}$ are shown in Figure 6. It can be seen that, in the whole state of change, with the increase of $g_{1}$ and $g_{2}$, the chaos of the system gradually intensified, from the steady state into the times cycle state, and finally into the chaos.

Figure 7 shows the system's variation of the cycle as $g_{1}$ and $g_{2}$ change from another angle. The different colors in Figure 7 represent the different periods of the system at this time. It can be seen that as the adjustment speed increases, the number of system cycles increases, and finally the chaotic state appears.

We get Figures 8(a) and 8(b) which show the average diagram of the profits of the manufacture and the third-party with respect to $g_{1}$ and $g_{2}$. From the two pictures we can find that the profits of the manufacture and the third-party are stable when $g_{2}$ and $g_{1}$ are small. And with the generation of the bifurcation, the system gradually steps into the chaos and both profit levels have showed a downward trend.

3.2. Largest Lyapunov Exponent. When the adjustment speed of the decision variable in the system changes, the largest Lyapunov exponent will have dynamic variation, so we can analyze the dynamic characteristics of the system through the largest Lyapunov exponent. If the largest Lyapunov exponent is less than 0 , the system is in a stable state; if the largest Lyapunov exponent is equal to 0 , the system is in a periodic motion or quasi periodic motion; if the largest Lyapunov exponent is greater than 0 , the system is in a chaotic state.

Figure 9(a) shows the largest Lyapunov exponent when $g_{2}$ is fixed at 0.01 and $g_{1}$ changes from 0.22 to 0.4 ; Figure $9(\mathrm{~b})$ shows the largest Lyapunov exponent when $g_{1}$ is fixed at 0.22 and $g_{2}$ changes from 0.45 to 0.587 . Comparing the largest Lyapunov exponent and the bifurcation diagram shown in 
Figure 8 , it can be seen that the values of $g_{1}$ and $g_{2}$ when bifurcation occurs and gets in chaos are consistent with the bifurcation diagram shown in Figure 8.

3.3. Kolmogorov Entropy. When the adjustment speed of the decision variable in the system changes, the Kolmogorov entropy also will have dynamic variation, so we can study the evolution of the system through the change of entropy.

Figure 10(a) shows the Kolmogorov entropy when $g_{2}$ is fixed at 0.01 and $g_{1}$ changes from 0.22 to 0.4 ; Figure $10(\mathrm{~b})$ shows the Kolmogorov entropy when $g_{1}$ is fixed at 0.22 and $g_{2}$ changes from 0.45 to 0.587 . Comparing the Kolmogorov entropy and the bifurcation diagram shown in Figure 9, it can be seen that the entropy of the system is increasing rapidly when bifurcation occurs and gets in chaos. However, when the system reenters the cycle state again, the entropy will appear a certain degree of decline.

3.4. Chaotic Attractor. When the system is in a stable state, attractor will be a stable fixed point; when the system goes into a chaotic state, attractor will occupy a larger space and the structure of the chaotic attractor will be more complicated. When $g_{1}=0.22, g_{2}=0.4$, the system is stable due to the bifurcation diagram, the chaotic attractor in this condition is shown in Figure 11.

When $g_{1}=0.4, g_{2}=0.01, g_{1}=0.22$, and $g_{2}=$ 0.58 , the system is in a chaotic state based on the bifurcation diagram shown in Figure 11; the chaotic attractors in these two conditions are shown in Figures 12(a) and 12(b).

From Figures 3-12, it can be seen that, in a closedloop supply chain with dual-channel recycling, the faster the adjustment of the decision variables in the system is, the more responsive the enterprise is and the more easily the market will get into chaos. According to the information theory, when the system is stable and in order, the probability of optimal price information will be pretty large and the information entropy will be low; when the system is chaotic, the information of the decision variables is out of order and the information entropy will be high. When the initial value of decision variable is fixed, the equation will not change with the adjustment speed; however, the velocity can affect the period of the market from order to chaos. Therefore, in a closed-loop supply chain with a manufacturer and a thirdparty recycling competitively, both the manufacturer and the third-party should make their decisions with an overall consideration about the market situation and competitor's response rather than adjust their recycling prices over quickly and blindly.

3.5. Sensitive Dependence on Initial Conditions. When $g_{1}=$ $0.22, g_{2}=0.4$, the system stays stable according to the three judgment methods above. The initial values of the recycling prices $p_{m}$ and $p_{t}$ are both taken 1 and 1.01. After multiiterations, the differences between the two sets of numerical solutions are shown in Figure 13. We can find that, in the beginning iterations, there is a little difference, but after about 15 times iterations, the difference gradually reduces to zero.
Thus it can be seen that chaotic system is very sensitive to initial value; just like the butterfly effect, a small difference in initial value can cause a huge deviation after multiepisodes. This gives us a revelation that decision makers should choose the initial value of their decision variables more prudently.

\section{Chaos Control}

From the previous discussion, chaotic phenomenon is unstable and initially sensitive, which will always be harmful to the economic market. Therefore, we should take measures to prevent chaos before it occurs and control chaos after it has occurred. In this paper, parameter control method is used to control chaos.

The discrete dynamic system which has not been controlled can be expressed as

$$
\begin{aligned}
& p_{m}(t+1)=f_{1}\left(p_{m}(t), p_{t}(t)\right) \\
& p_{t}(t+1)=f_{2}\left(p_{m}(t), p_{t}(t)\right) .
\end{aligned}
$$

After being controlled by parameter adjustment, the controlled system can be written as

$$
\begin{aligned}
p_{m}(t+1) & =(1-u) f_{1}\left(p_{m}(t), p_{t}(t)\right)+u p_{m}(t) \\
p_{t}(t+1) & =(1-u) f_{2}\left(p_{m}(t), p_{t}(t)\right)+u p_{t}(t) .
\end{aligned}
$$

We can discuss the influence of control parameter $u$ on the chaotic system by numerical simulation. When $g_{1}=0.4$, $g_{2}=0.01$, the system is in a chaotic state from the analysis above. After adding $u$, the variation diagram of the recycling price $p_{m}$ and $p_{t}$ with change of the control parameter $u$ is shown in Figure 11. It can be seen that when $u=0$, the whole system is in a chaotic state and the two variables are both uncontrollable. As $u$ increases, the two variables gradually go from chaos, via double-periodic state, to a stable state finally. It means that the entropy decreases as $u$ increases, and the chaos is controlled effectively.

Figures 14(a) and 14(b) indicate the variation of recycling prices $p_{m}$ and $p_{t}$ under chaos control. It is obvious that when control parameter $u>0.25$, the system comes to stable statue and keeps in it. As shown in the equation of controlled system, it is time to use feedback control and parameter adjustment when the system is out of control and even falls in chaos, which enables the system to go back to stability from harmful chaotic state. From theoretical perspective, the controlled system equation describes control process as the chaos points gradually converge to the Nash equilibrium points.

On the other hand, chaos control benefits recycling market management. As we know, recycling market is in its infancy, and it is hard to be managed for lacking of perfect recycling system. If there exits chaos in the system, it can be explained as a result of malicious price competition among channels, which damages the ordering of fresh established recycling market. In consequence, chaos control is of vital importance to recycling market. This paper introduces methods of state feedback control and parameter adjustment to control the chaos that occurs in the repeated game process by suppressing or delay chaos. 


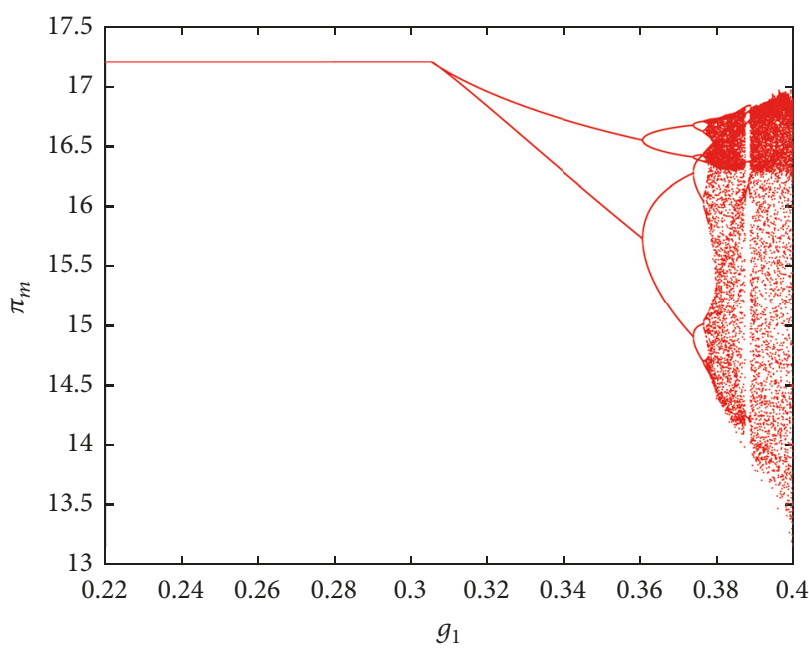

(a)

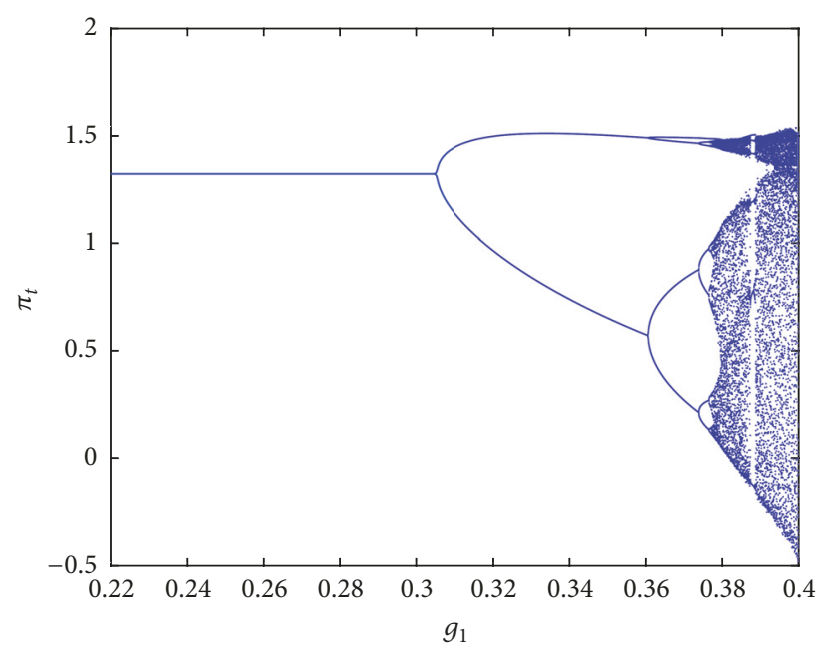

(b)

Figure 3: (a) Bifurcation diagram of $\pi_{M}$ with respect to $g_{1}$ when $g_{2}=0.01$, (b) bifurcation diagram of $\pi_{T}$ with respect to $g_{1}$ when $g_{2}=0.01$.

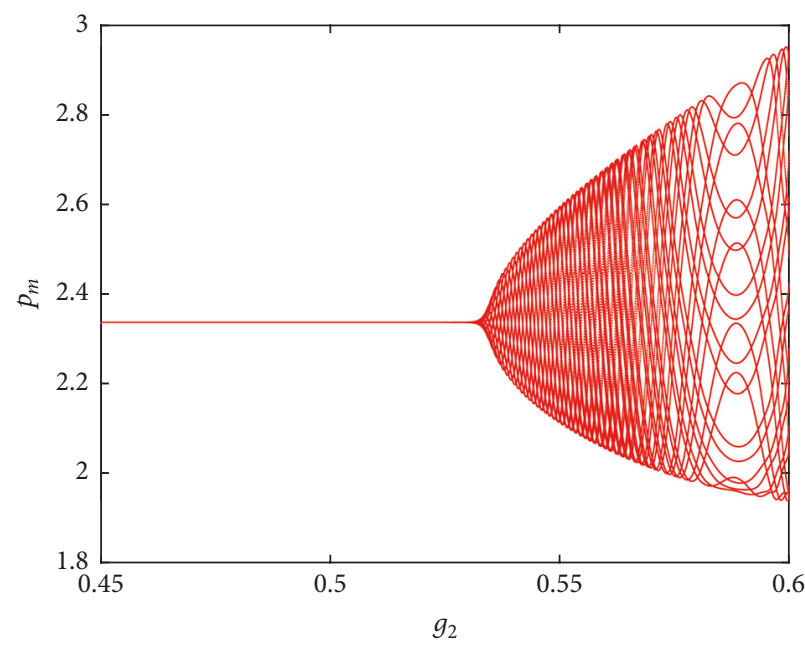

(a)

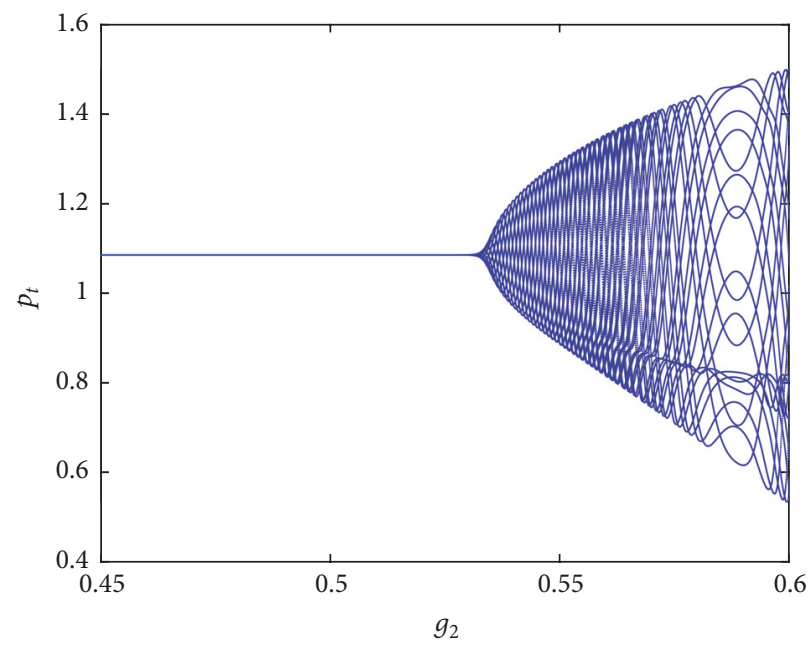

(b)

FiguRE 4: (a) Bifurcation diagram of $p_{m}$ with respect to $g_{2}$ when $g_{1}=0.22$, (b) bifurcation diagram of $p_{t}$ with respect to $g_{2}$ when $g_{1}=0.22$.

Both the manufacturer and the third-party hope that the market is stable, because they can make decisions more easily and pursue the maximum profits steadily. However, the closed-loop supply chain market is complicated; the change of the decision variables may lead the market from stability to chaos. Hence the manufacturer needs to be cooperative with the third-party and take some measures in order to delay or eliminate chaos to the benefit of the market developing and stability.

\section{Conclusion}

In this paper, we build a closed-loop supply chain model according to real market situation with dual-channel recycling composed of a manufacturer and a third-party. Considering that the consumer perception towards the remanufactures products is uncertain and limited compared to the new products, we introduce a parameter $d$ to show the quantity of remanufactured products consumers who are willing to buy in a period cycle. We analyzed the dynamic game model and try to find the condition that keeps the system stable. Using MATLAB to perform numerical simulation, we discussed the dynamic characteristics of the system by bifurcation diagram, Largest Lyapunov exponent, and chaotic attractors. We analyze the system from the perspective of the information entropy. We also analyzed the initial value sensitivity, which is a feature of the chaotic system. Finally, we introduced an efficient method to control chaos. We get the following conclusions:

(1) If the manufacturer and the third-party adjust their decision variables too quickly, the entropy of the system will increase; the system will go into a chaotic state. In chaos, the manufacturer and the third-party 


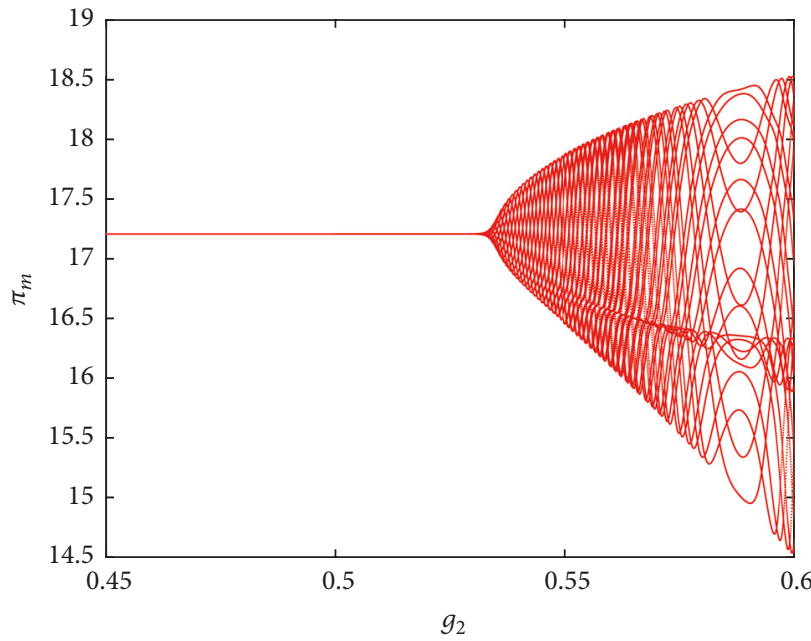

(a)

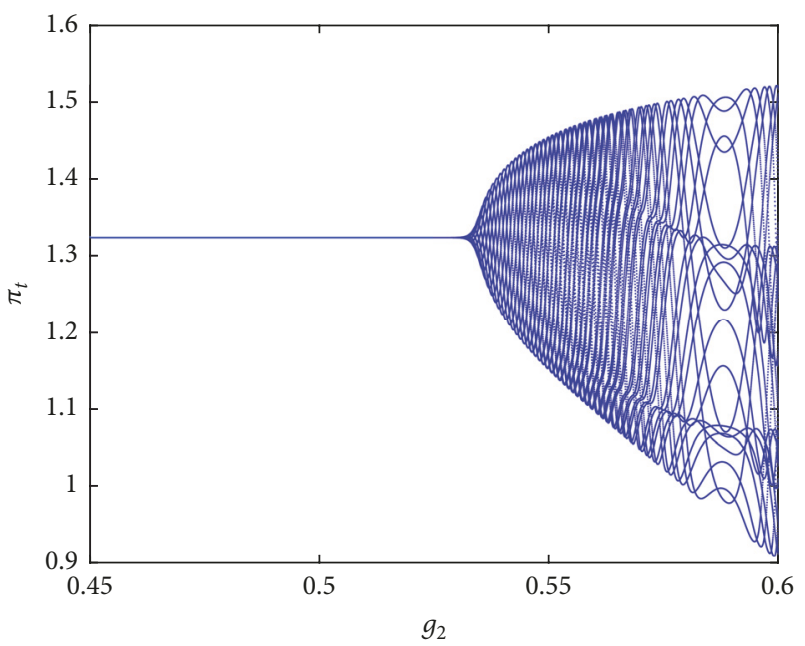

(b)

Figure 5: (a) Bifurcation diagram of $\pi_{M}$ with respect to $g_{2}$ when $g_{1}=0.22$, (b) bifurcation diagram of $\pi_{T}$ with respect to $g_{2}$ when $g_{1}=0.22$.

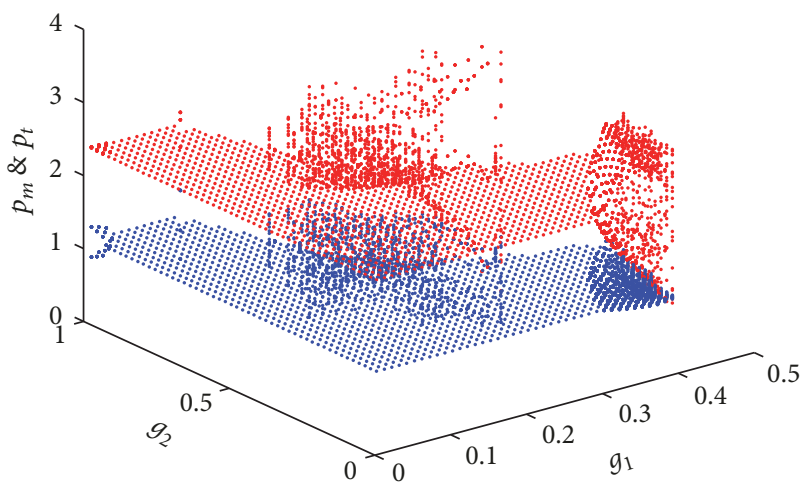

Figure 6: 3D Bifurcation diagram of $p_{m}$ and $p_{t}$.

are so difficult to predict their prices and profits that they cannot acquire steady profits to meet the market demand. According to the information theory, when the system is in a stable state, the information is in order and has more value which means that the entropy is low; when the system is in chaos, the large uncertainty of variable information will lead to the result that more additional information is needed to make it clear and the entropy is large.

(2) When the system is in a stable state, the difference value of the decision variables because of the initial value's subtle change will gradually become zero as the iterations increase. When the system is in a chaotic state, the difference value of the decision variables because of the initial value's subtle change will enlarge by hundreds of times after several iterations.

(3) The chaotic system can be delayed or eliminated effectively by adding a adjustment parameter, so the manufacturer and the third-party should cooperate with each other to take some measures to prevent and control chaos. We believe that this conclusion

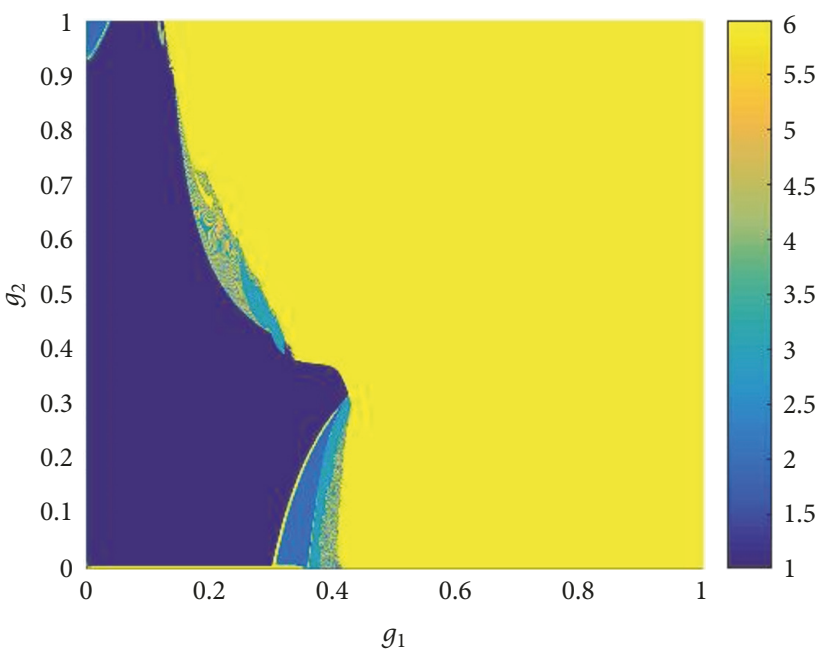

Figure 7: 2D Bifurcation diagram of $p_{m}$ and $p_{t}$.

has not only theoretical direction but also practical significance.

At the same time, we believe that in order to better improve the model and further contact to the actual situation, this article can be further explored in the following areas:

(1) Further increase the number of manufacturers which participate in the game, although a small number of oligopolies occupy a large share in real life, but a small number of small recyclers which provide professional services may also bring a certain impact on the traditional market.

(2) Establish a cooperation mechanism between the game sides to maximize the collective interests of the game, and the establishment of the corresponding supply chain coordination mechanism. 


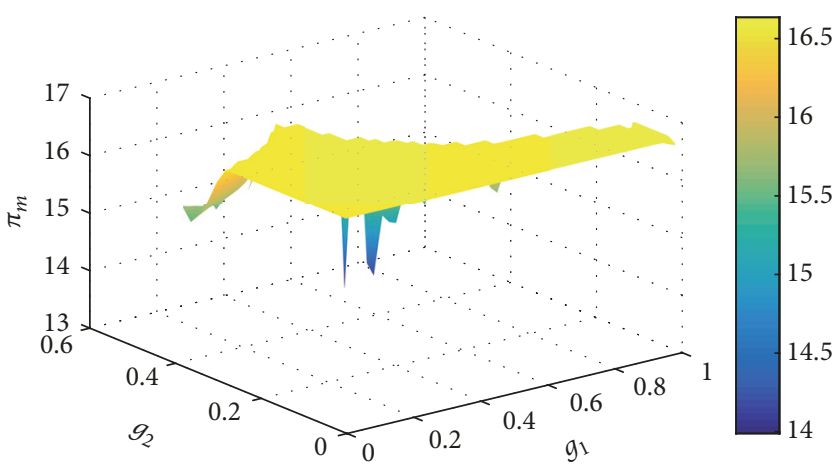

(a)

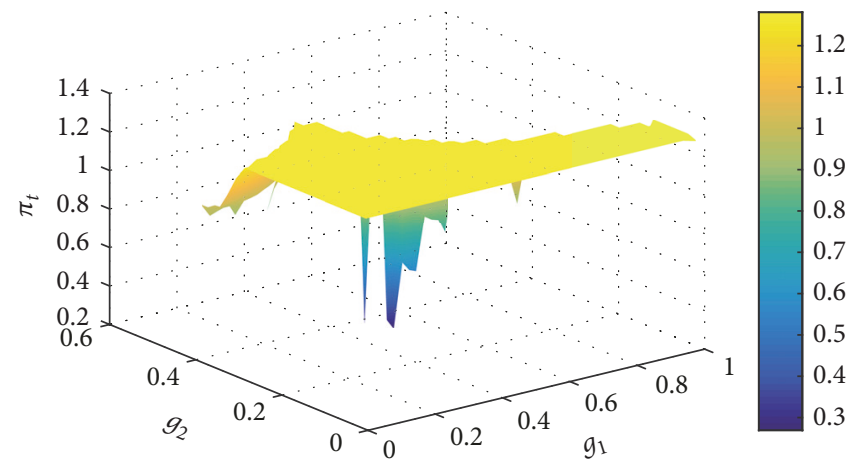

(b)

FIGURE 8: (a) Average profit diagram of $\pi_{M}$ with respect to $g_{2}$ and $g_{1}$, (b) average profit diagram of $\pi_{T}$ with respect to $g_{2}$ and $g_{1}$.

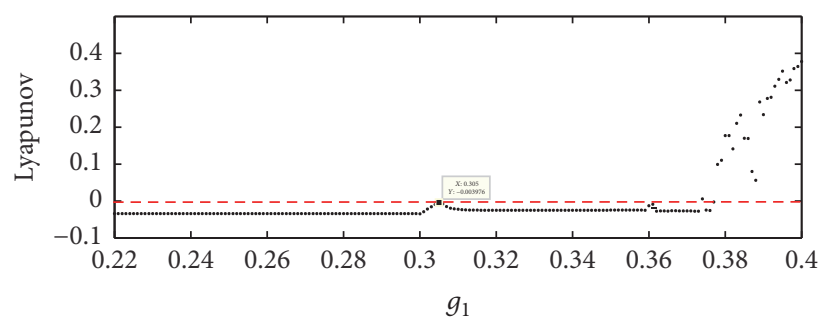

(a)

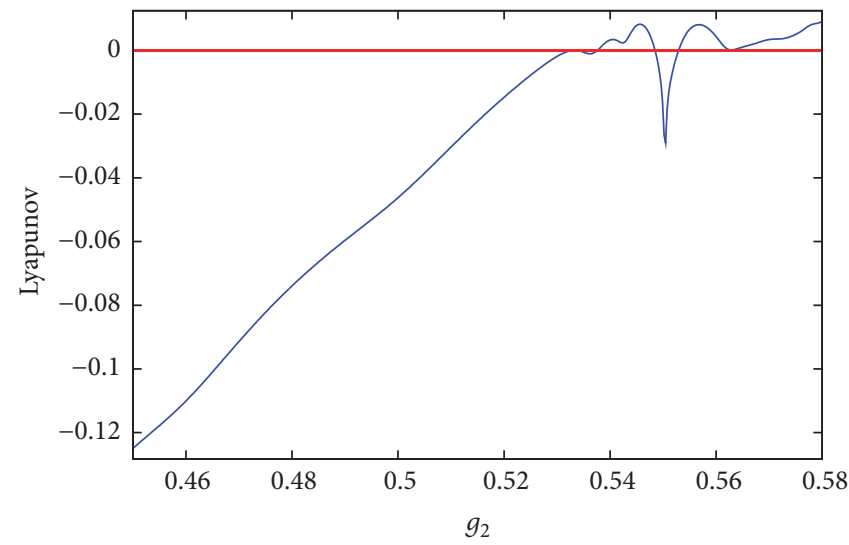

(b)

FiguRE 9: (a) Largest Lyapunov exponent with respect to $g_{1}$ when $g_{2}=0.01$, (b) largest Lyapunov exponent with respect to $g_{2}$ when $g_{1}=0.22$. 


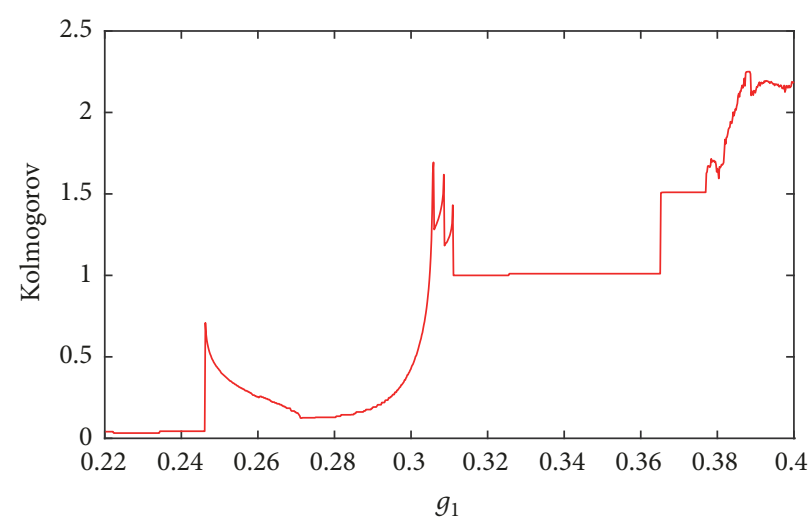

(a)

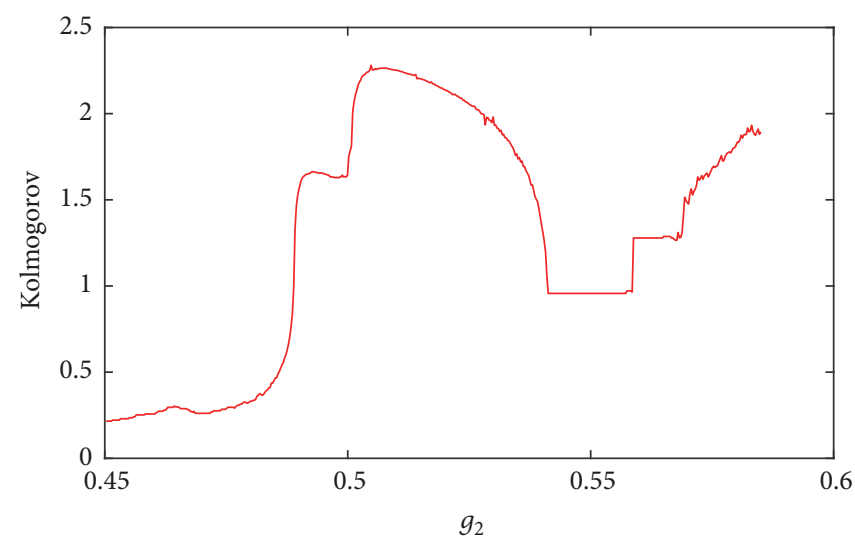

(b)

FIGURE 10: (a) Kolmogorov entropy with respect to $g_{1}$ when $g_{2}=0.01$, (b) Kolmogorov entropy with respect to $g_{2}$ when $g_{1}=0.22$.

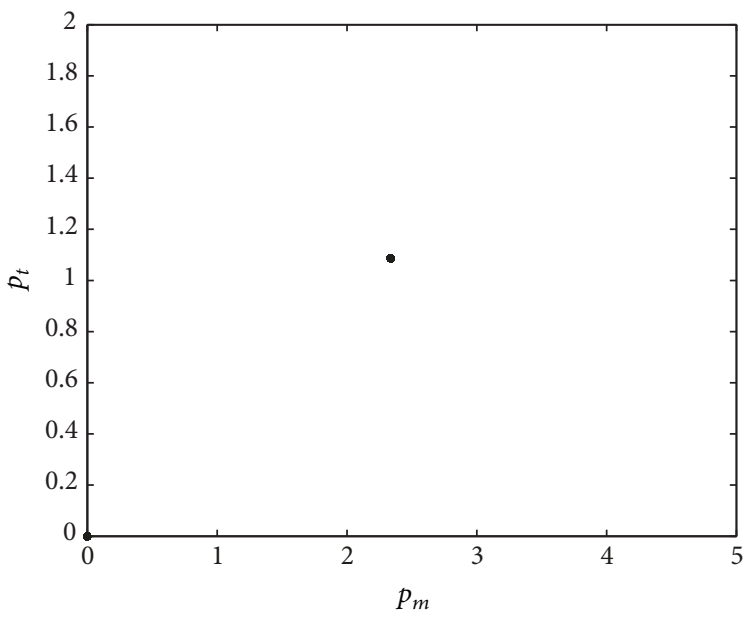

FIGURE 11: Chaotic attractor of the system when $g_{1}=0.22, g_{2}=0.4$.

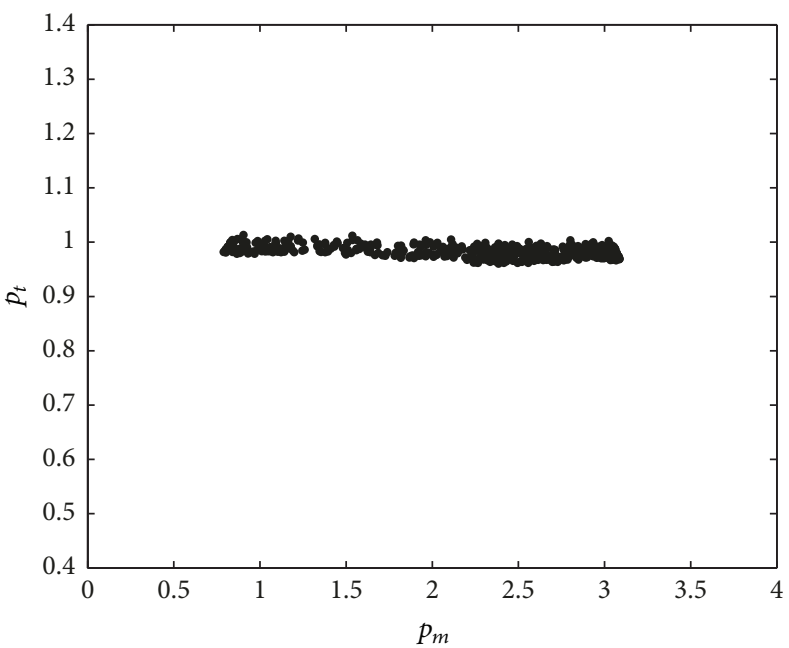

(a)

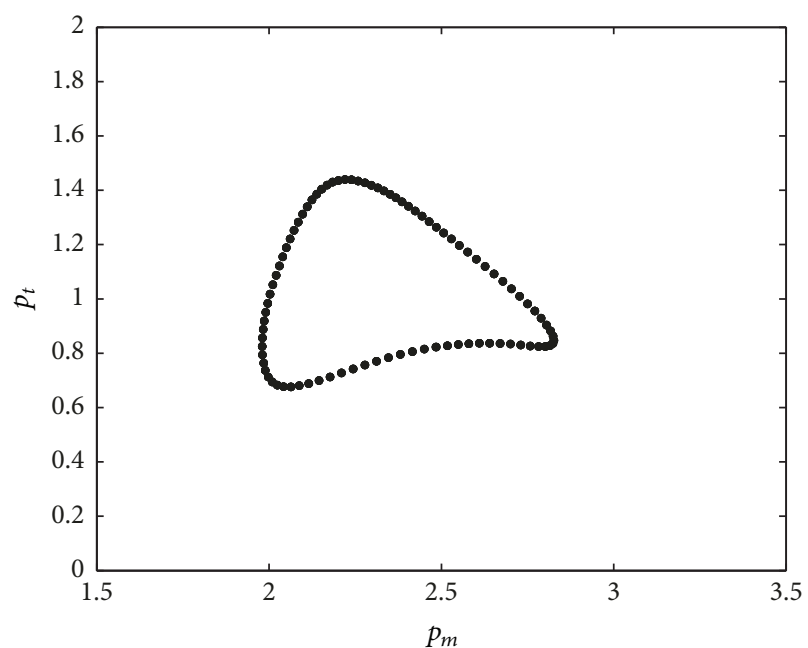

(b)

Figure 12: (a) Chaotic attractor of the system when $g_{1}=0.4, g_{2}=0.01$, (b) chaotic attractor of the system when $g_{1}=0.22, g_{2}=0.58$. 


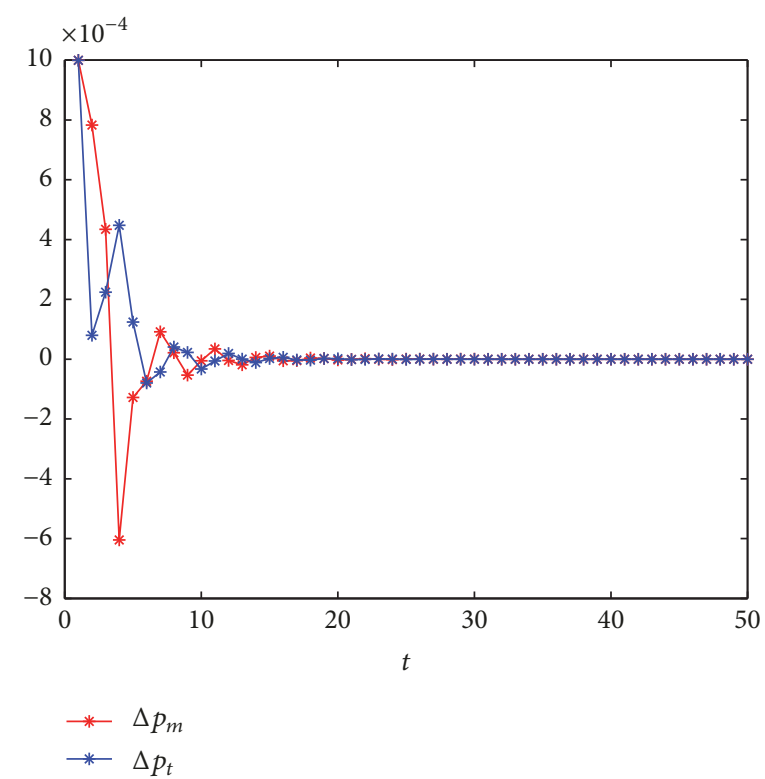

FIGURE 13: Initial value sensitivity of recycling price in stable system when $g_{1}=0.22, g_{2}=0.4$.

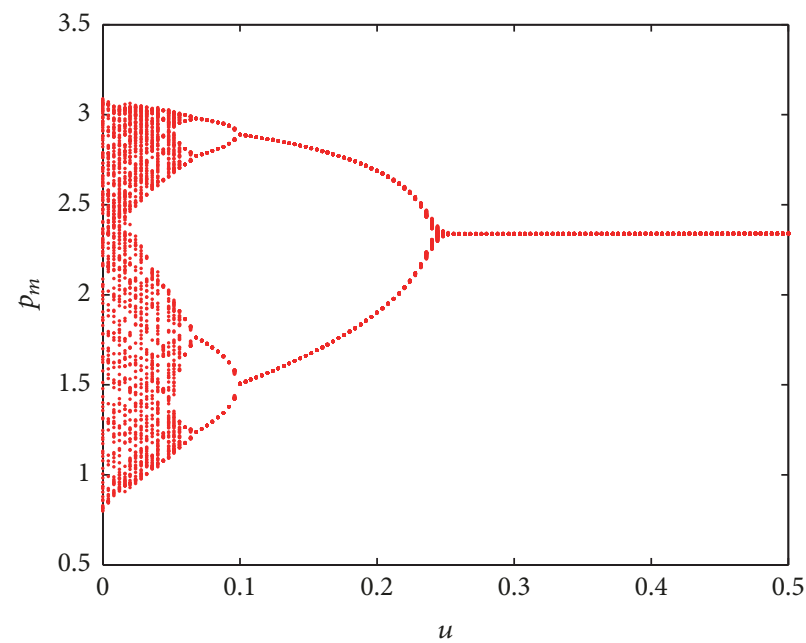

(a)

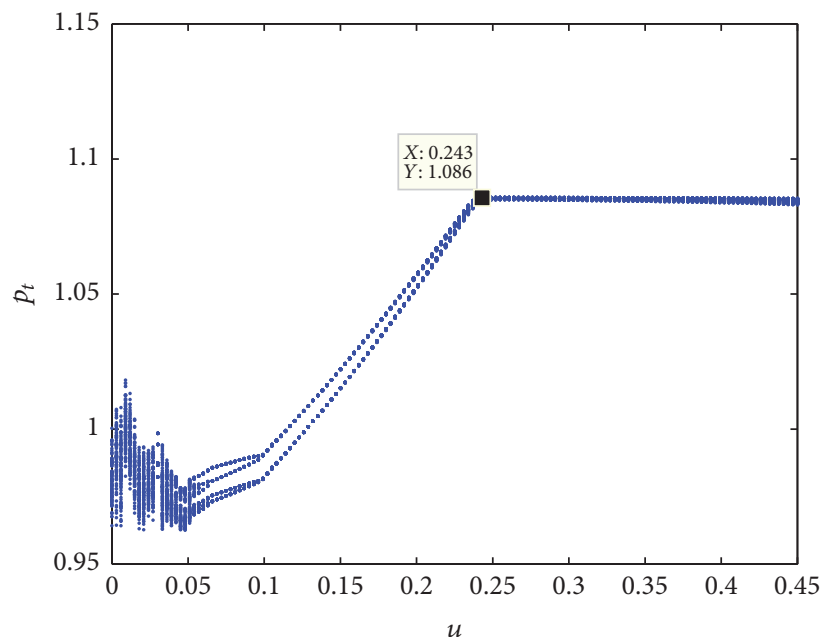

(b)

FIGURE 14: (a) (b) Variation diagram of the recycling prices $p_{m}$ and $p_{t}$ with change of the control parameter $u$ when $g_{1}=0.4 g_{2}=0.01$. 
(3) When the recovery work has made some progress and sufficient data are available, a more accurate numerical fit can be made.

\section{Conflicts of Interest}

The authors declare no conflicts of interest.

\section{Acknowledgments}

The research was supported by the National Natural Science Foundation of China (no. 71571131).

\section{References}

[1] Y. Liang, S. Pokharel, and G. H. Lim, "Pricing used products for remanufacturing," European Journal of Operational Research, vol. 193, no. 2, pp. 390-395, 2009.

[2] M. Ketzenberg, "The value of information in a capacitated closed loop supply chain," European Journal of Operational Research, vol. 198, no. 2, pp. 491-503, 2009.

[3] M. I. G. Salema, A. P. Barbosa-Povoa, and A. Q. Novais, "Simultaneous design and planning of supply chains with reverse flows: a generic modelling framework," European Journal of Operational Research, vol. 203, no. 2, pp. 336-349, 2010.

[4] G. Kannan, P. Sasikumar, and K. Devika, "A genetic algorithm approach for solving a closed loop supply chain model: a case of battery recycling," Applied Mathematical Modelling: Simulation and Computation for Engineering and Environmental Systems, vol. 34, no. 3, pp. 655-670, 2010.

[5] J.-B. Sheu and A. Y. Lin, "Hierarchical facility network planning model for global logistics network configurations," Applied Mathematical Modelling: Simulation and Computation for Engineering and Environmental Systems, vol. 36, no. 7, pp. 30533066, 2012.

[6] J.-C. Lu, Y.-C. Tsao, C. Charoensiriwath, and M. Dong, "Dynamic decision-making in a two-stage supply chain with repeated transactions," International Journal of Production Economics, vol. 137, no. 2, pp. 211-225, 2012.

[7] J.-M. Chen and C.-I. Chang, "The co-opetitive strategy of a closed-loop supply chain with remanufacturing," Transportation Research Part E: Logistics and Transportation Review, vol. 48, no. 2, pp. 387-400, 2012.

[8] M. Huang, M. Song, L. H. Lee, and W. K. Ching, "Analysis for strategy of closed-loop supply chain with dual recycling channel," International Journal of Production Economics, vol. 144, no. 2, pp. 510-520, 2013.

[9] T. Maiti and B. C. Giri, "A closed loop supply chain under retail price and product quality dependent demand," Journal of Manufacturing Systems, vol. 37, pp. 624-637, 2015.

[10] Y. He, "Acquisition pricing and remanufacturing decisions in a closed-loop supply chain," International Journal of Production Economics, vol. 163, pp. 48-60, 2015.

[11] M. Zu-Jun, N. Zhang, Y. Dai, and S. Hu, "Managing channel profits of different cooperative models in closed-loop supply chains," Omega , vol. 59, pp. 251-262, 2016.

[12] A. A. Elsadany, "Dynamics of a delayed duopoly game with bounded rationality," Mathematical and Computer Modelling, vol. 52, no. 9-10, pp. 1479-1489, 2010.
[13] Y. Guo and J. Ma, "Research on game model and complexity of retailer collecting and selling in closed-loop supply chain," Applied Mathematical Modelling: Simulation and Computation for Engineering and Environmental Systems, vol. 37, no. 7, pp. 5047-5058, 2013.

[14] J. Ma and Y. Guo, "Research on third-party collecting game model with competition in closed-loop supply chain based on complex systems theory," Abstract and Applied Analysis, vol. 2014, Article ID 750179, 22 pages, 2014.

[15] L. Xie and J. Ma, "The dangers of precipitous adjustment speed of recovery price to the closed-loop supply chain and improvement measure," WSEAS Transactions on Mathematics, vol. 13, pp. 1-11, 2014.

[16] J. Ma and F. Si, "Complex dynamics of a continuous Bertrand duopoly game model with two-stage delay," Entropy, vol. 18, no. 7, p. 266, 2016.

[17] Z. Han, J. Ma, F. Si et al., "Entropy complexity and stability of a nonlinear dynamic game model with two delays," Entropy, vol. 18, no. 9, p. 317, 2016. 


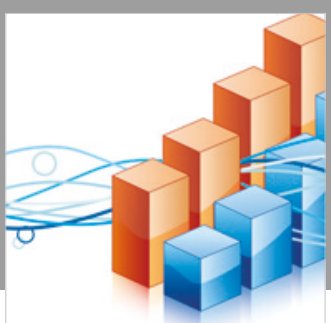

Advances in

Operations Research

\section{-n-m}
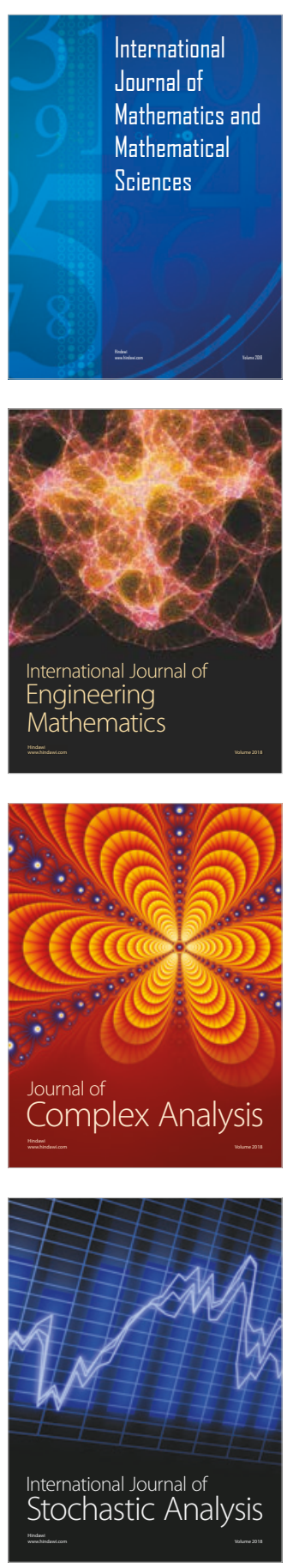
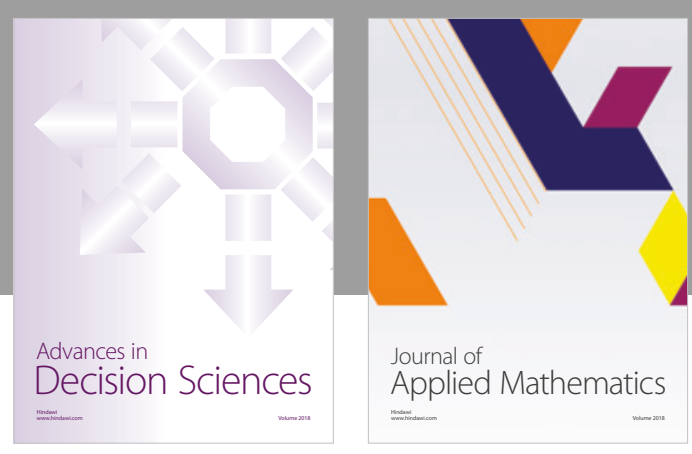

Journal of

Applied Mathematics
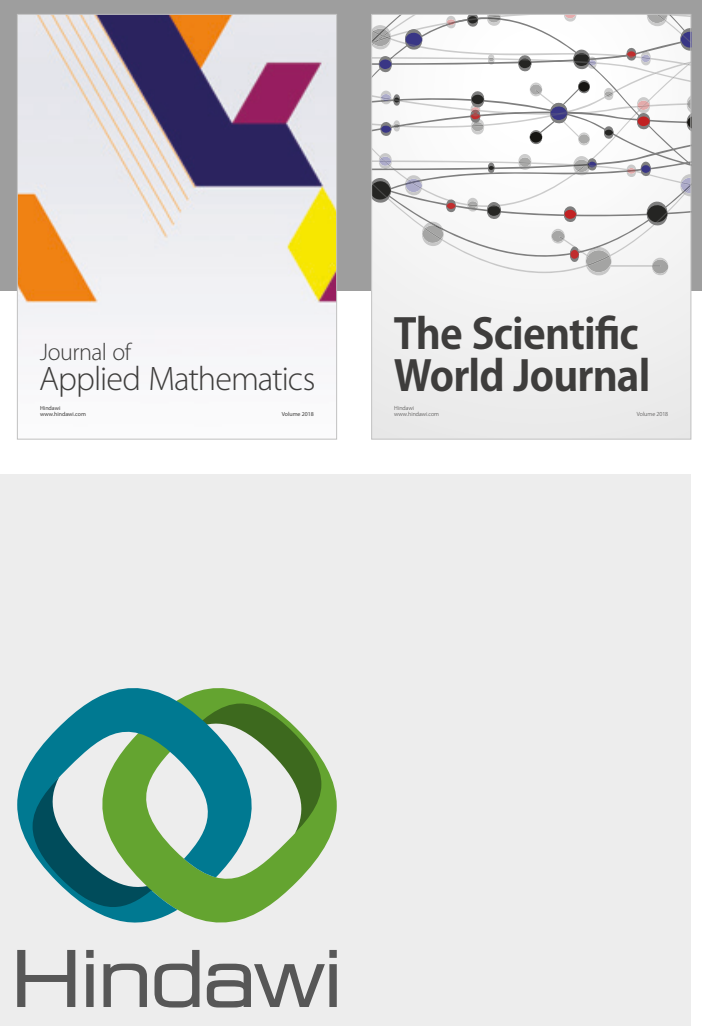

Submit your manuscripts at

www.hindawi.com

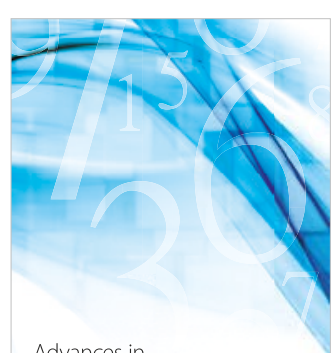

Advances in
Numerical Analysis
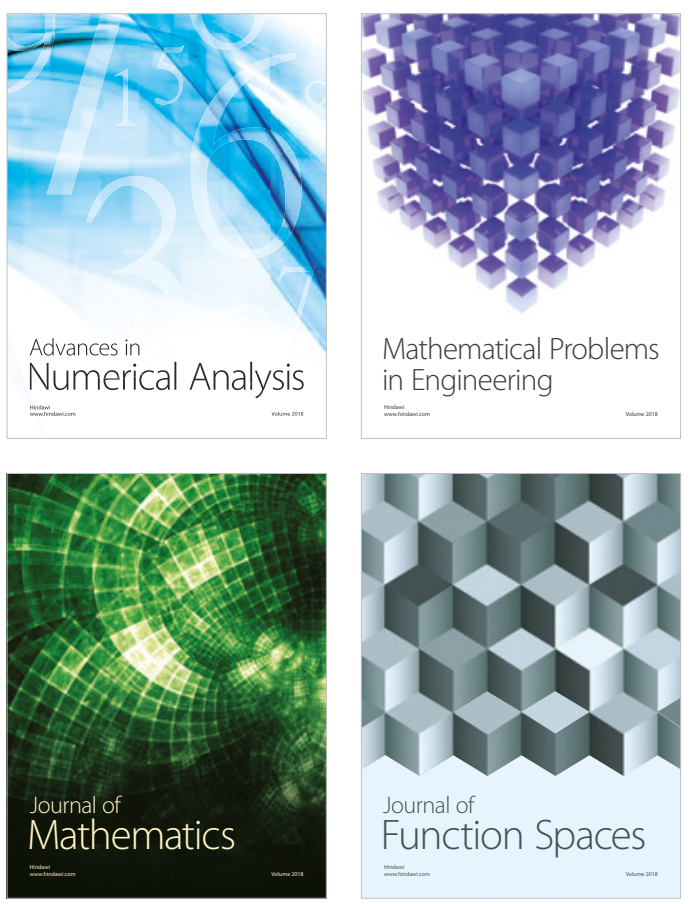

Mathematical Problems in Engineering

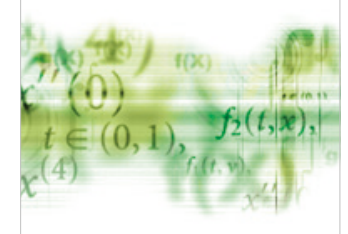

International Journal of

Differential Equations

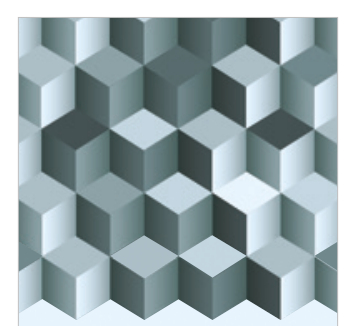

Journal of

Function Spaces
The Scientific

World Journal

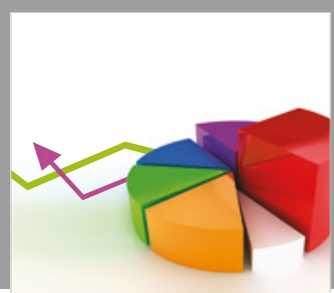

Journal of

Probability and Statistics
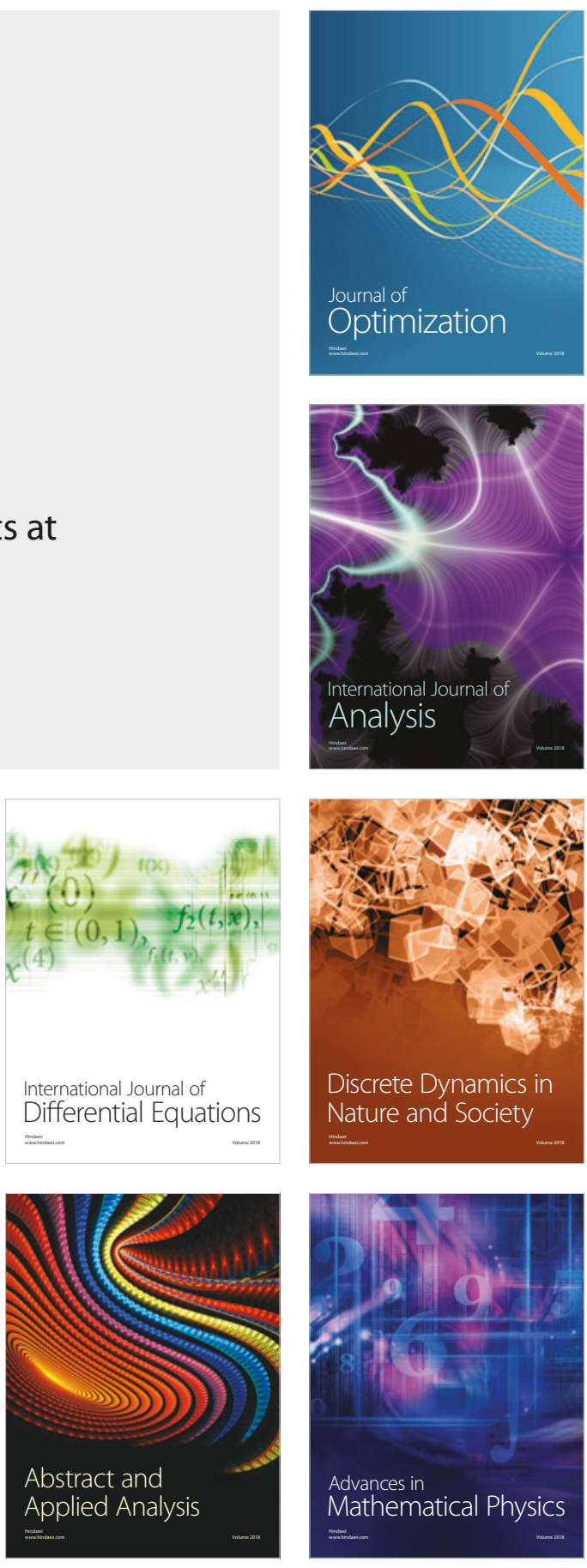